\title{
Synthesis and in vitro bioactivity evaluation of new glucose and xylitol ester derivatives of 5- aminosalicylic acid
}

\begin{abstract}
New glucose and xylitol esters of 5-amino salicylic acid (5-ASA) were synthesized followed by evaluation of their in vitro antimicrobial, anti-cancer and anti-inflammatory activities. The results of the antimicrobial activity assessment revealed that the new final esters were more effective against Gram-negative as well as Gram-positive bacteria than the original drug. Furthermore, the new final products were confirmed by a cytotoxicity assay over HT-29 and $3 \mathrm{~T} 3$ cell lines to be less toxic for normal cells compared to the initial drug. On the other hand, however, their suppressive effect against cancerous cells was somewhat lower. Meanwhile, the anti-inflammatory activity assay over a RAW264.7 macrophage cell line demonstrated that the NO inhibition activity of the conjugated drug to the previously mentioned saccharides, especially to glucose, has slightly improved compared to the non-conjugated drug. Finally, in silico screening was also performed in order to predict the potential interactions and binding energy of the novel products against cyclooxygenase (COX-1/COX2) and lipoxygenase (5-LOX) proteins. Findings indicated that the new products had greater hydrogen bonds and binding affinities with the active sites of proteins towards 5-ASA.
\end{abstract}

